\section{ON THE CONVERSION OF RADIANT ENERGY INTO SONOROUS VIBRATIONS ${ }^{1}$}

MESSRS. GRAHAM BELL and Sumner Tainter (American Association for the Advancement of Science, Boston, August 27, 1880) have shown that under certain conditions intense rays of light, if allowed to fall with periodic intermit. tence upon thin disks of almost every hard substance, will set up disturbances in those disks corresponding to this periodicity which result in sonorous vibrations. Mr. Bell (Fournal of the Society of Telegraph Engineers, December 8, I880) has subsequently shown that such effects are not confined to hard substances, but that they can be produced by matter in a liquid or gaseous form.

These discoveries have elicited a considerable amount of in terest, and have led to the inquiry whether the sonorous effects are due, as the discoverers themselves surmised, to light. or as the president of the Royal Society, Prof. Tyndall, and others have suggested, to radiant heat.

Messrs. Bell and Tainter have partially answered this question by showing that the disturbances are not necessarily due to light, for they found that sheets of hard rubber or ebonite-a substance opaque to light-do not entirely cut off the sounds, but allow certain rays to pass through, which continue the effect. $M$. Mercadier, who has studied the subject with great care (Comptes rendus, December 6, 1880), lias shown that the effects are confined to the red and ultra-red rays. Moreover Mr. Bell has shown that gases, such as. sulphuric ether, which Prof. Tyndall has proved to be highly absorbent of heat rays, while they are transparent to light rays, are remarkably sensitive to this intermittent action. Dr. Tyndall has more recently read a paper before the Society (Proc. Roy. Soc., January 3, 188I) proving that these sonorous effects are a function of all gases and vapours absorbing radiant heat, and that the intensity of the sounds produced is a measure of this absorption.

The negative proof of Messrs. Bell and Tainter can be rendered positive if it can be shown that ebonite is diathermanous.

By very careful experiments made upon the diathermancy of different materials, ebonite proved to be as diathermanons as rock-salt.

It is therefore clear that the sonorous vibrations of Bell and Tainter are the result of disturbances produced by some thermic action rather than by any luminous effect.

Now the questions arise, Is this thermic action expansion and contraction of the mass due to its absorption of heat? Or is it a disturbance due to molecular pressure similar to that which produces the rotation of the radiometer? Or is it due to some other cause?

The argument against the first assumption when applied to hard disks is that time is a material element in such actions, and that the rate of cooling of warmed diaphragms is too slow to admit of such effects. Lord Rayleigh (NATURE, January zo, 188r) has questioned the validity of this argument, and has shown that if the radiating power of the body experimented on were sufficiently high a slow rate of cooling would be favourable to rapid fluctuations of temperature. It became desirable to test this point experimentally. Very delicate apparatus was constructed for the pur ose.

Heat from various sources and at various distances, from bright lime-light to dull heat from hot metallic surfaces, was allowed to fall through rotating vanes intermittently on different bodies; but notwithstanding every precaution, and the many materials used, not more than six interruptions per second could be produced, although the system was beautifully sensitive to the smallest changes of temperature.

It was evident from these experiments that the sonorous effects of hard disks could not be explained by the change of volume due to the impact of heat rays and their absorption by the mass of the disk.

Is the action then due to molecular pressure similar to that which produces the rotation of the radiometer?

It is quite true that the radiometer effect is one visible only in very high exhaustions, but Mr. Crookes (Phil. Trans., 1878, Part i., $\$ 220$ ) detected "the existence of molecular pressure when radiation falls on a black surface in air of normal density."

Whenever radiant energy falls on an absorbent surface in air, such as a disk of blackened wood, its wave-length is degraded

I Abstract of a paper by Mr. William Henry Preece, read at the Royal Society, March ro. or lowered, and it is converted in thermometric heat. The molecules of air striking this warmed surface acquire heat, and move away from it with increased velocity, and as action and reaction are always alike in moving away, they give the body a "kick." Since there is no such action on the other side of the disk, there is a difference of pressure between the two sides which gives it a tendency to move away from the source of energy. The effect is very much smaller in air at ordinary pressures than in air at a very low vacuum, because in the former case the mean free path of the molecules is very small, and the rebounding molecules help to keep back the more slowly approaching molecules. Nevertheless, molecular pressure is experienced, and if of sufficient magnitude and rapidity, it ought to produce sonorous vibrations. It seemed probable that the element of time does not enter here so prominently as in the previous case, for the radiometer effect is a mere surface action of the disk, and not one affecting its mass. Hence it was hoped that the retarding effects would be eliminated. If the sonorous action, therefore, be due to a radiometer action, a difference of effect would be observed if the side of a disk exposed to the source of energy be either blackened by lampblack or camphor carbon, or if it be polished or whitened.

An apparatus was constructed similar in principle to that described by Messrs. Bell and Tainter.

An ebonite disk well blackened on one side when exposed to the intermittent rays was found to produce sounds, while a similar ebonite disk equally well-whitened, gave slightly less intense sounds. A zinc disk blackened gave weak sounds, while a similar disk polished gave sounds much weaker. A mica disk blackened gave scarcely any sounds at all, while a clean mica disk gave slightly better sounds.

These effects were produced many times and on different occasions, and they were so unsatisfactory as to throw doubts on the accuracy of the radiometer explanation. They were not so decided as theory led one to anticipate. The effects produced by the zinc disk, though very weak, favoured the theory; those given by the mica disk completely refuted it; wbile those given by the ebonite disks were almost of a neutral character.

The question occurred whether in Bell and Tainter's experiments the disks vibrated at all.

A delicate microphone was fixed in various ways on the case holding the disks. Although the sounds emitted in the hearingtube were quite loud, scarcely any perceptible effect was detected on the microphone. Had the disk sensibly vibrated, its vibrations must have been taken up by the case. A microphone never fails to take up and magnify the minutest mechanical disturbances.

It was thus evident that the disk did not play a prime part in this phenomenon, but that the result might be due wholly to an expansion and contraction of the air contained in the air space behind the disk.

With a new clean case and an ebonite disk the sonorous effects were feeble, but if a lens were placed close in front of the ebonite disk, so as to make a second air space in front of the disk, the sonorous effects were magnified considerahly.

The ebonite disk was fitted with an extremely delicate micro phone, which in this case gave good indications upon the telephone, but whether the vibrations were the results of the vibrations of the disk itself, or of the air in which the microphone was placed, was doubtful.

If the lens were removed and the disk left supported without any air cavity, either behind or in front of it, no perceptible sound could be obtained, proving that the effects were really due to the vibrations of the confined air, and not to those of the disk. It was therefore determined to dispense with the disk altogether. The disk was therefore removed, the lens remaining; the sonorous effects were nil.

Another case was taken under similar circumstances, i.e. without the disk, but the effects were very loud-- 60 ; in fact, the best results which had yet been obtained. Now the only difference between the one case or cup and the other was that the one was blacked in the interior, and the other was not.

Hence the former case was again taken without the disk, and though when clean it gave no effect, when its interior was blacked by camphor smoke, it gave sounds as strong as the loudest effect yet produced. It was thus evident that the sonorous effects were materially assisted by coating the sides of the containing vessel with a highly-absorbent substance, such as the carbon deposited by burning camphor. It remained to be seen how far the lens played a part in this phenomenon. 
The lens was now removed from the front of the case, and it was replaced by a movable glass plate ( $x \cdot 5$ millims. thick); the sounds were the same, but they gradually ceased on gradually uncovering the front opening of the case, so as to give the air room to expand.

The glass plate was replaced by a heavy rigid plate of rock-salt 13 millims. thick, and the sounds were equally loud. The plate was replaced by white note-paper. The sounds were very faint, but perceptible. It was replaced by thin cardboard, and the effect was nil.

Hence it is abundantly evident that these sonorous vibrations are due to the motions of the contained air, and not to those of the disk; that they are actually improved by the removal of the disk; that their production is materially assisted by lining the surface of the containing space with an absorbent substance that they are dependent on the heat rays that pass through; and that they disappear when the rays are cut off from the air cavity by an athermanous diaphragm.

Dr. Tyndall having shown in the paper already referred to, that water vapour responded actively to these intermittent actions, a clean empty one-ounce glass flask was taken and exposed to the intermittent beams. No sound was produced.

It was then filled with water-vapour by pouring a small quantity of water into it, and warming it in a flame; fair sounds were the result.

The flask was filled with the dense smoke from burning camphor, and the sounds were intensified considerably.

Another clear one-ounce glass flask was taken. When clear no sounds were heard. When filled with tobacco-smoke fair sounds, but when filled with heavy camphor smoke very loud sounds were obtained. One side of the flask was blackened on the outside, the other side remaining clear. On exposing the clear side to the light fair sounds were obtained, but on exposing the blackened side, no sound's were produced. The flask was blackened in the interior on one side only. When the blackened side was near the source fair sounds, and when it was away from the source better sounds were heard. When the flask was cleaned all sounds disappeared. A thin glass plate was now blackened on one side and placed in front of the case. When the black surface was outside no sounds were obtained. When the black surface was inside good sounds were the result. When the glass was cleaned the sounds became still better. An ebonite plate was similarly treated. When the blackened surface was outside fair sounds were obtained. When the blackened surface was inside very poor sounds were the result.

This being an anomalous result, several experiments were now made to test the behaviour of opaque and transparent bodies, when used as disks, for while in the previous experiments the effect was greatest when the blackened surface faced the interior, here we find the opposite result produced, viz., the greatest effect was produced when the blackened surface was on the exterior.

Several experiments were then made, from which it appeared that transparent bodies behave in an opposite way to opaque bodies. Glass and mica can be rendered athermanous and silent by making the carbon deposit sufficiently thick. Zinc, copper, and ebonite can produce sonorous effects by a proper disposition of carbon. The effect in these latter cases may be due either to molecular pressure, in fact to a radiometer effect, though very feeble in intensity ; or it may be the result of conduction through the mass of the diaphragm, that is, radiant heat is reduced to thermometric heat by absorption by the carbon deposit on the outside of the disk ; and this heat is transmitted through the disk and radiated to the absorbent gases in the interior.

Several experiments were made which fully establish the in. ference that the effect is one of conduction, and that the blackened surface of an opaque body like zinc acts as though the source of heat were transferred to the outside surface of the disk.

Tubes of various sizes and dimensions were now tried to confirm Messrs. Bell and Tainter's observations on tubes. They invariably gave out satisfactory sounds when the intermittent rays were directed into the interior of the tubes, which were always considerably intensified by blackening their interiors and closing the open end with a glass plate.

It was shown that there is a time element, and that the loudness of the note emitted depends upon the rapidity with which the contained air not only absorbs the degraded energy, but upon the rapidity with which it gives up its heat to the sides of the case and the exits open to it. Though the pitch of the maxi- mum note varied with the cavity and the amount of radiant heat transmitted, its quality never varied, notwithstanding the great diversity of materials used as diaphragms.

Since these sonorous effects are due to the expansions of absorbent gases under the influence of heat, and since wires are heated by the transference of electric currents through them, it seemed possible that if we inclosed a spiral of fine platinum wire in a dark cavity, well blacked on the inside, and sent through it by means of the wheel-break, rapid intermittent currents of electricity from a battery, heat would be radiated, the air would expand, and sounds would result. This was done, and the sounds produced were excellent. In fact, with four bichromate cells sounds more intense than any previously observed were obtained.

Furthermore it was evident that if the wheel-break were replaced by a good microphone transmitter, articulate speech should be heard. This was done, and an excellent telephone receiver was the consequence, by means of which speech was perfectly reproduced.

The explanation of these remarkable phenomena is now abundantly clear.

It is purely an effect of radiant heat, and it is essentially one due to the changes of volume in vapours and gases produced by the degradation and absorption of this heat in a confined space. The disks in Bell and Tainter's experiments must be diathermanous, and the better their character in this respect the greater the effect; remove them, and the effect is greater still. Messrs. Bell and Tainter (Fournal of Society of Telegraph Engineers, December 8, 1880) showed that the sounds maintained their timbre and pitch notwithstanding variation in the substance of the disk, and M. Mercadier found that a split or cracked plate acted as well as when it was whole. These facts are consistent with the expansion of the contained air, but not with any mechanical disturbance of the disks. Moreover M. Mercadier showed that the effect was improved by lampblack, but he applied it in the wrong place.

The disks may, and perhaps do, under certain conditions, vibrate, but this wibration is feeble and quite a secondary action. The sides of the containing vessel mnst possess the power to reduce the incident rays to thermometric heat, amd impart it to the vapour they confine, and the more their powerin this respect, as when blackened by carbon, the greater the effect. The back of the disk may alone act in this respect. Cigars, chips of wood, smoke, or any absorbent surfaces placed inside a closed transparent vessel will, by first absorbing and then radiating heat rays to the confined gas, produce sonorous vibrations.

The heat is dissipated in the energy of sonorous vibrations. In all cases, time enters as an element, and the maximum effect depends on the diathermancy of the exposed side of the cavity, on its dimensions and surfaces, and on the absorbent character of the contained gas.

\section{THE EARTHQUAKE IN ISCHIA}

THE Island of Ischia, the Pithecusa of the ancients, is some twenty miles in circumference, and appears'to be the continuation of the north-western boundary of the Gulf of Naples It consists of an old volcanic mountain sloping down on all side to the sea. The southern rim of the old crater has been remove by denudation, leaving the northern as a curved serrated ridge, forming the peak of Monte Epomeo.

Situated on the southern slopes of the island are only a few and unimportant villages.

Going from east to west along the northern slope we have first the capital Ischia, then we encounter the great trachytic lava stream which issues laterally from the slope of Epomeo, and after a course of two miles entered the sea, forming a promontory. This is the so.called lava Del' Arso, of A.D. 1302 Next are encountered two very fresh-looking craters, from which lava streams have flowed. Then we come to Casamicciola, a small town of about 4000 inhabitants, to the north-west of which is the village of Lacco Ameno. At the eastern end of the island is the town of Forio.

The district in which are situated Casamicciola and Lacco is thus bounded on the north by the sea, on the south by the ridge of Monte Epomeo, on the west by a spur stretching from the latter into the sea, forming the Punta Cornacchia, and on the east are the two hills called Mont $\in$ Rotaro and Montagnone, the new-looking craters already spoken of. 